\title{
Flood Prediction Using IoT Enabled Sensor Network and Machine Learning
}

\author{
Sneha Suresh ${ }^{1}$, Meenu Mathews ${ }^{2}$, Anju KS ${ }^{3}$, Lincymol Abraham ${ }^{4}$, Dr.Vinodh P Vijayan ${ }^{5}$ \\ ${ }^{1}$ Department of Computer Science, India,snehasureshchry@ gmail.com \\ ${ }^{2}$ Department of Computer Science, India,meenupandarakappil@gmail.com \\ ${ }^{3}$ Department of Computer Science, India,anjukaladharan2012@gmail.com \\ ${ }^{4}$ Department of Computer Science, India,lincyabraham888@ gmail.com \\ ${ }^{5} \mathrm{Head}$ of the Department of Computer Science,India, vinodh.pvijayan@mangalam.in
}

\begin{abstract}
Floods are one of the most common natural disasters that affects humanity. Unless we take the proper precautions, it will endanger human life itself. Flood prediction using IoT enabled sensor network and machine learning outlines a very efficient way to properly understand and manage floods. This technology can be used to evaluate the risk of flooding, there by speeding up precautionary measures. IoT is a network of computing devices with unique identifiers which helps transferring data across the devices without interaction between human and computers. By using different sensors, information about various environmental conditions were collected and transfer it to google sheet with the help of IoT technology. The client can analyse the data in the form of dataset from a remote device and predict the risk of flood.
\end{abstract}

Key words: Classification, Decision tree, IoT-Internet of Things, Machine Learning

\section{INTRODUCTION}

Flood is a natural hazard that causes many losses in every country in the world. To some extent, it is possible to monitor the weather using various technologies, but it requires accurate real-time monitoring of data to cope up with sudden flooding .Since floods are most destructive and has far more impact on human life than other natural disasters, it is a challenge for the government to find the best way to deal with it. There were systems built to monitor floods that integrate rainfall data, water level and flow data from various geo-sensor networks and is brought together in a web-based platform that disseminates information to the public [2]. The requirements of realtime data and knowledge-intensive stages of automation and supervision deals with the uncertain, unfrozen, heuristic and fuzzy information [10].

Flood monitoring and prediction models have significant importance for risk assessment and management. So, an efficient prediction model will contributes a lots to monitoring, analysing and prevention strategies of government. It introduces an approach which helps to predict the risk of flooding in a very cost effective, easy and robust way. Flood prediction using IoT enabled sensor network and machine learning introduces a new way of predicting the chance of flood in an easy and efficient way by the integration of IoT and machine learning technologies.

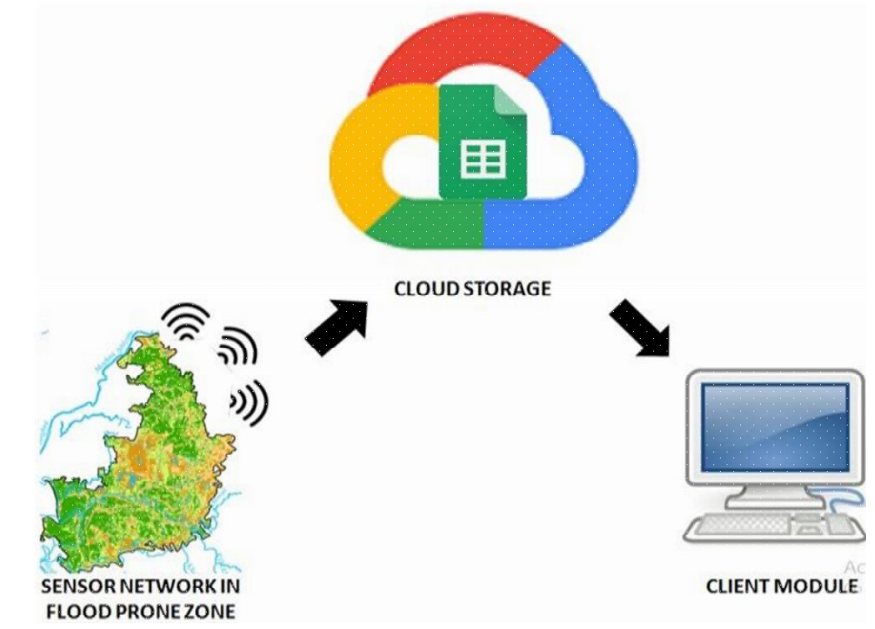

Figure 1:Sensor based centralized flood detection

\section{LITERATURE REVIEW}

Flood monitoring, prediction and forecasting is most important in nowadays. There are many question arises related to the flooding problems. It is the urgent need of the today's world to survive from the flooding. With the aid of computational methods has been studied and introduced different methods and technologies to predict, monitor and forecast the flood.

M. S. Baharum, R. A. Awang and N. H. Baba [1] provide a flood monitoring and alerting system to monitor the flood in different water resources. They used Graphical User Interface (GUI) display to monitor and Short Messaging Services (SMS) Gateway for sending alerts by Visual Basic 6.0 and Ubuntu Server Edition. The system is aimed to 
monitor the water level using water sensors which are placed in different low lying areas either in normal dangerous conditions. They divided the system into different levels such as normal zone, level 1, level 2, level 3 and dangerous zone. The data from the sensors are collected is analysed and sends alerts to the user throughGlobal System for Mobile communication (GSM). It is cost effective and efficient data measurement can be done. But the system may cause problems of inaccurate data from the sensors, faults in sending alerts. Ribeiro Alexandra, Cardoso Alberto, Marques

Alfeu S, Simoes Nuno E [2] discuss the case study, the stretch of the Portuguese Mondego River between the upstream dam system Aguieira - Raiva - Fronhas and the downstream Dam Bridge of Coimbra City. For the web based platform the communication between client and server done through AJAX (Asynchronous JavaScript and XML) and prototype implementation is based on the DHI MIKE OPERATIONS framework .They provide a web based platform for river flood monitoring by collecting different information such as water levels, flows and rainfall by geo-sensor networks. The measurements are downloaded and represent as maps using GIS. The platform can effectively monitor the water level in a region only. Swapnil Bande and Prof. Dr. Viranda V. Shete [6] introduced a flood prediction system which is based on IoT and neural networks. ANN is used to predict flood. They measured temperature, pressure, rainfall, humidity, river water level using sensors and to find the temporal correlative information for flood analysis. It enhances the scalability and reliability of flood management system. But the use of $\mathrm{Wi}-\mathrm{Fi}$, there will be a chance of connection lost or any other disruptions and the cost is high.

Mohammed Khalaf, AbirJaafarHussain, Dhiya Al-Jumeily, Paul Fergus and Ibrahim OlatunjiIdowu [7] proposed the system which employed by machine learning algorithm. Data from the sensors are taken and analyses it. Based on the analysed data, it sends alerts as sms to the users by using GSM technology. Accuracy of the system is much better. But it is not easy to track the equipment that located in various field such as riverbank or low-lying area. Indra Riyanto, Lestari Margatama, Angga Ariawan, Luhur Bayuaji, Mia Rizkinia, Dodi Sudiana, Harry Sudibyo S and Josaphat Tetuko Sri Sumantyo [8] introduced the flood warning system which is a web camera sensor coupled with Lidar data. The water level image is taken using a web camera and the resultant image is represented as flood map. .They segmented the flood related area from Lidar data at $1 \mathrm{~m}$ intervals and the water level is recorded at $10 \mathrm{~cm}$ intervals. It is efficient to display flooded areas when the river rise. The images taken may be inappropriate, inaccurate and lead to some faults in sending alerts to the users.

\section{SYSTEM ARCHITECTURE}

The proposed system mainly consist of three modules:

\section{a.Sensor Module \\ b.Cloud Storage \\ c.Client System Module}

Sensor module includes mainly five sensors to monitor temperature, humidity, soil moisture, water level and rainfall. To improve the system performance more sensors can be included to the module easily. All these sensors are directly connected to ESP8266 NodeMCU which is an IoT platform. The data signals received from the sensors are clustered and transfers to the drive (as google sheets). In the drive, client obtains data in the form of data sheets. So there is no need for further conversion. The client can directly download the data set for the application of Machine Learning algorithm to predict if there any chances of flood. Decision tree classificationalgorithm is used to predict the flood risk in the proposed system.

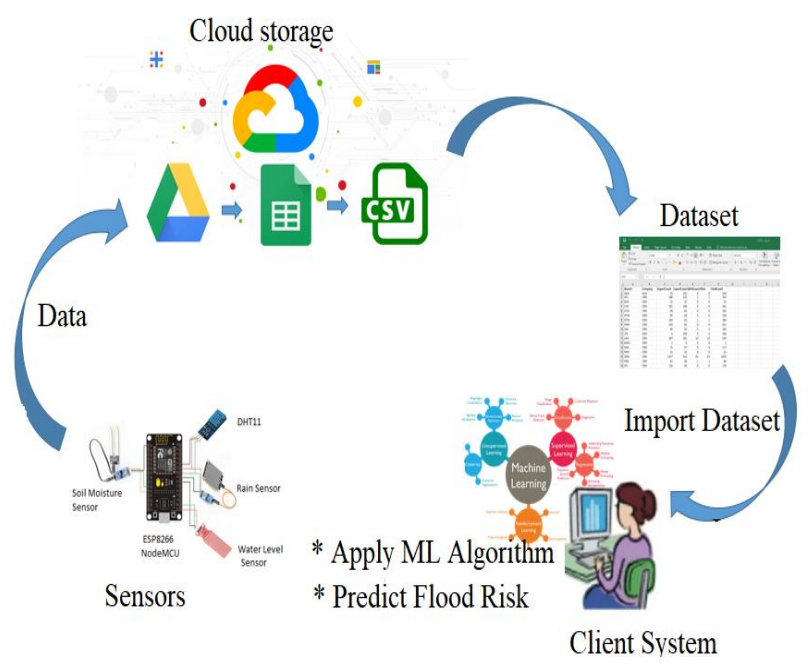

Figure 2: Flood Prediction System

\section{DETAILED FUNCTIONING}

\subsection{IoT Enabled Sensor Network}

The internet of things (IoT) is an interconnection of devices like sensors or actuators which transfers data and information over the internet with less human intervention. The internet and the devices are the two important parts of IoT. In short, it is the network of smart sensors and helps to reduce humans work load [3][4].Sensors are devices that collect information from the surroundings, by detecting the changes and transfer the data to other devices or a computer processor by different means. The WSN structures are integrated with the gateway are offered wireless 
connectivity that supports the wired globe and disseminated nodes. The energy of wireless sensor network offered with the capability of huge amount of data with minute nodes which are collected and organized for their transmission. Data are produced by the nodes are usually relay with the sink node across a multi hop network. Network coverage is one of the major estimation process in the wireless network. It is constantly beneficial for the capability of organizing the network over the improved physical region. This can considerably enlarge the system importance to the end user [11]. Sensors, which forms the base for IoT systems, connect to IoT network after the processing of the information. The converted output signal from the sensor act as input for the microcontroller.

For predicting the flood risk different dedicated sensors including the temperature and humidity sensor, soil moisture sensor, water level sensor and rain sensor and monitors the environmental conditions. NodeMCU is one of the low cost, open source IoT platform. All the sensors are connected with

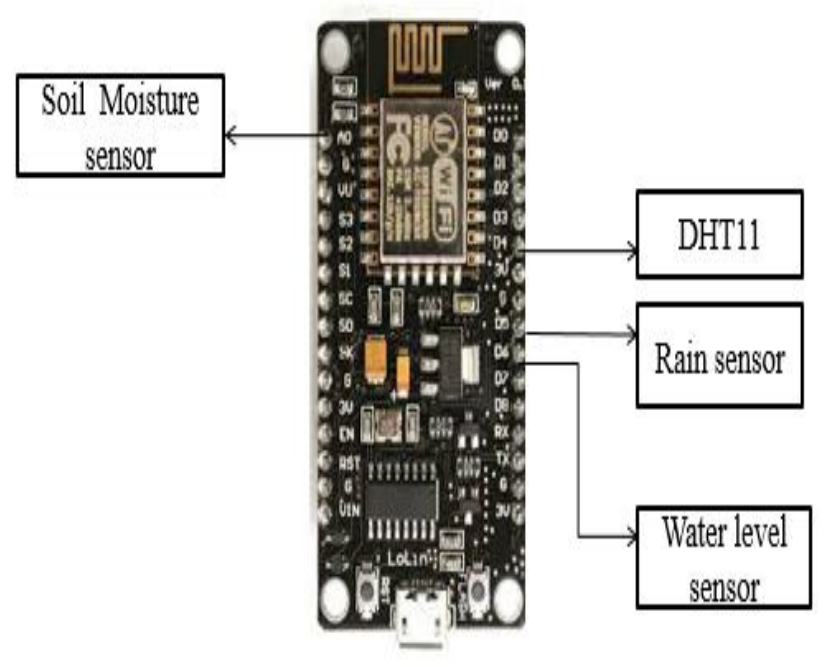

Figure 3: Connection of Sensors toESP8266 NodeMCU

ESP8266 NodeMCU. Using Wi-Fi module of NodeMCU the sensor data can be transfer into the drive.

- DHT11 Sensor: It is temperature and humidity sensor. This helps to measure the temperature and humidity in the environment and gives serial data as output and it can be easily interfaced with the microcontroller.

- Rain sensor: It is used to detect the amount of rainfall and helps to accurately predict flood risk.

- Water level sensor: Level of flowing water can be detected using this sensor.
- Soil moisture sensor: The moisture content in the soil can be determined using this sensor.

\subsection{Sensor to Google Sheet Data Transfer}

DHT11 sensor, Water level sensor, Rainfall sensor and Soil Moisture sensor are efficiently used to detect and collect the information about the changes in environmental conditions. The Water level sensors can be placed and arranged in such a manner that it would be triggered when water reaches a certain level thus detecting the current water level and it is compared with the previously collected dataset [1]. Rain water reaching the ground surface will infiltrate to the soil. Surface depressions and puddles begin to be filled with water when the soil reaches a point where in the rainfall intensity is greater than the infiltration capacity of the soil. In this stage, water begins to rise in level above the soil [5]. The soil moisture sensor senses the moisture content in the soil and gives the variations in the moisture content as values through it. The sensors are connected to the NodeMCU by using jumpers. The gathered data's from the sensors are processed

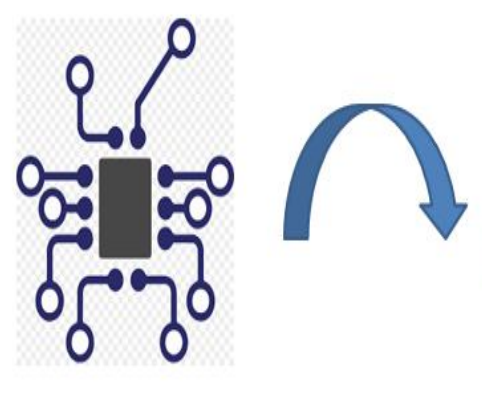

SENSOR NETWORK

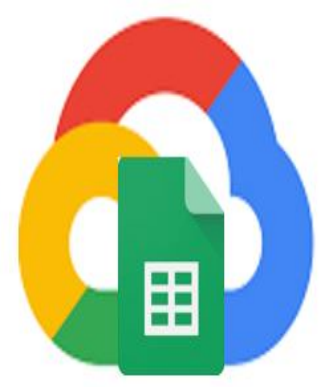

CLOUD STORAGE
Figure 4: Data Transfer from sensors.

and transferred to the google drive for prediction purposes. A time delay is sets for fetching the corresponding data from the sensors. So in the given time delay the data's are automatically uploaded into the google storage.

The microcontroller is programmed to transmit the serial output data from the sensors into different groups or clusters and the processed data is saved in the google drive. The data in the drive can be accessed from anywhere and the datasets in the google spreadsheet can be downloaded in the form of .csv file. It is possible to monitor the environmental conditions from a remote location, the only requirement is good internet connectivity. 


\subsection{Application of machine learning algorithm}

Machine learning is a part of artificial intelligence, where the computer system or machine learn from experiences (by analysing the patterns in the database). Different types of algorithms are using in machine learning technology for different purposes. Machine Learning process involves collecting all the data, cleaning the data and takes the appropriate data's that are needed for the problem solution.

Using the training data, machine learning algorithm can be trained in order to create a model. The machine learning algorithm is then applied to the input dataset to predict the output. Here, to predict the chance of flood the decision tree classification algorithm in machine learning is used. Decision tree uses supervised learning concept. Using decision tree algorithm the dataset is sampled into different clusters. The

data set using is the real time data from the sensors. Since the sensors produces a lot of data, introduction of delay in the circuit helps a limited amount of data to be stored in drive. Client can download the datasheet from the drive to test and train using decision tree algorithm. Decision tree model is best suited for this prediction.

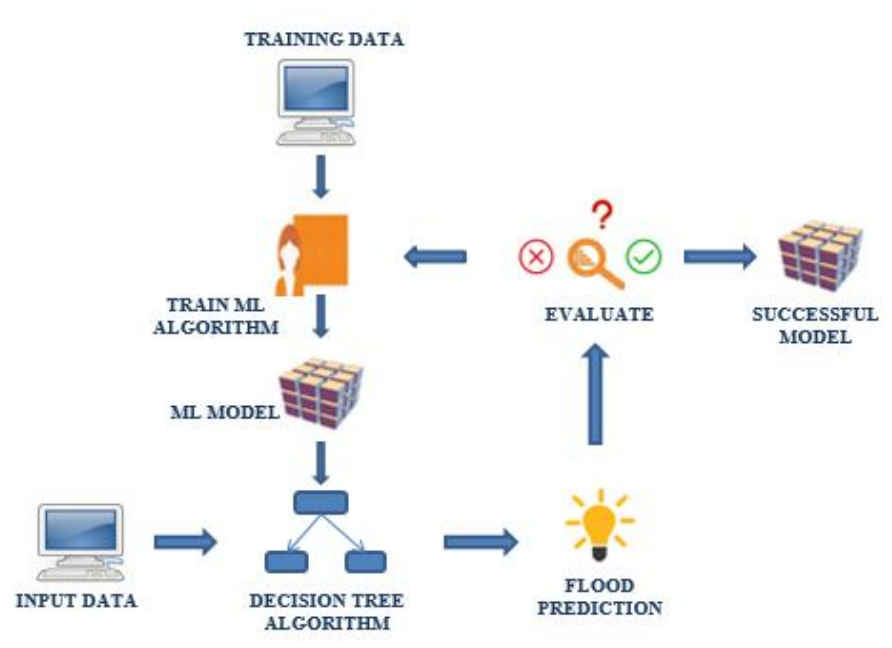

Figure 5: Machine Learning Process

\section{EXPERIMENTAL ANALYSIS AND RESULTS}

At first the sensors are placed in the flood prone area. Client can import drive-loaded sensor information into his system wherever he is. All it takes is good internet access. To download the data in the client system, the client must login to the page. If the user does not have an account, user can create an account and login with his own username and password. Client can convert sensor data into different clusters. Afterwards, user can download the dataset from the drive in the form of .csv file by applying machine learning algorithm the chance of flood can be predicted.

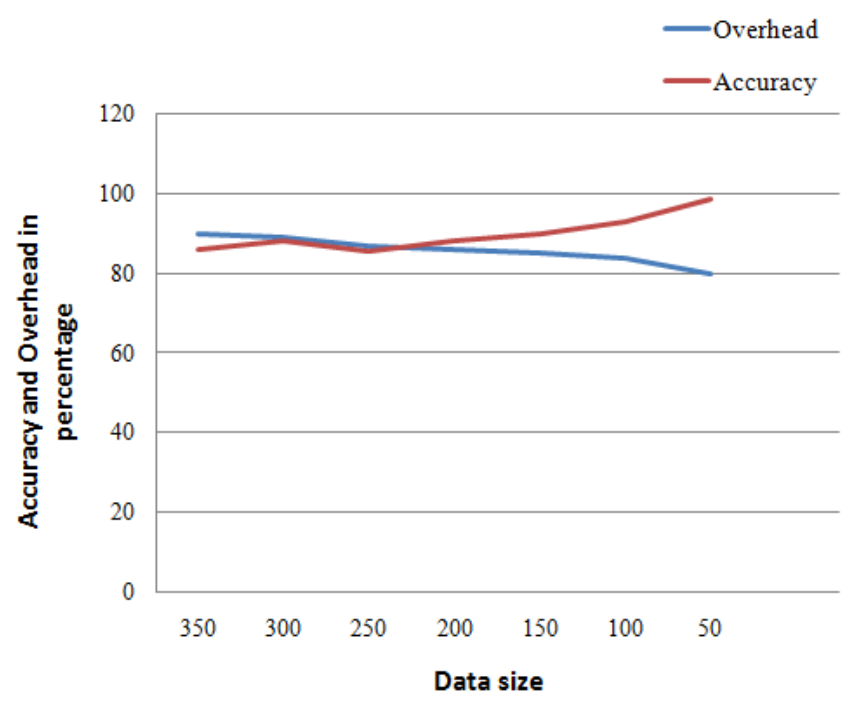

Figure 6: Classification

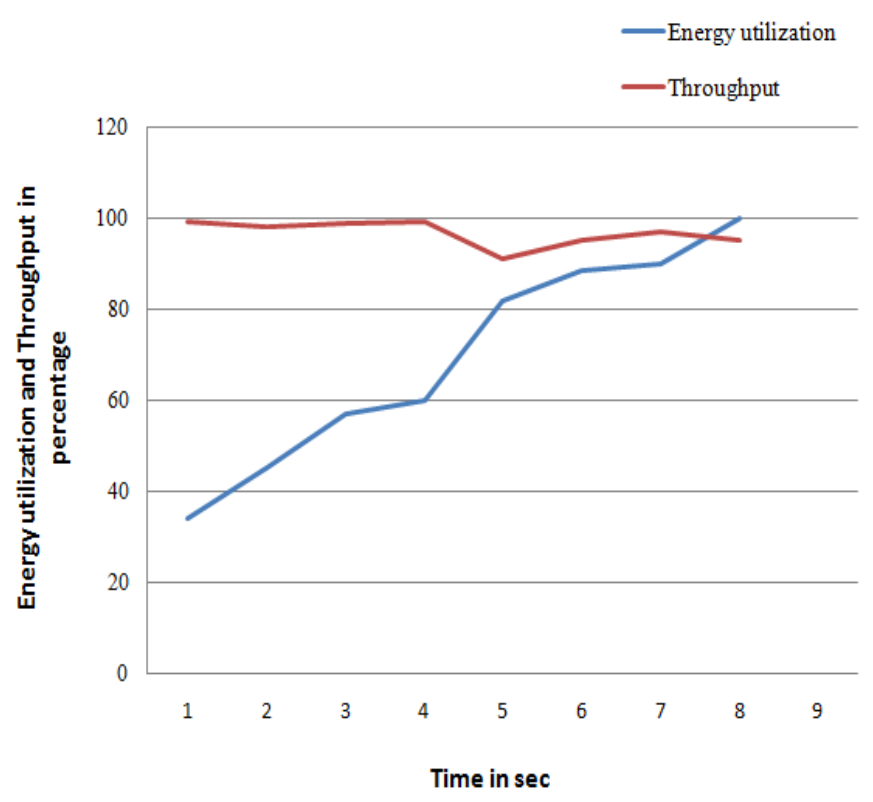

Figure 7: System performance

The graph is visualized using WEKA tool. Datasets are taken in .arff format (attribute relation file format).In the figure 6, classification overhead and accuracy is analysed. As the size of dataset increases accuracy increases and overhead decreases. Figure7 indicates the performance of the system. The energy utilization and throughput is analysed. As the time increases energy utilization increases. The throughput shows small deviations with respect to time. The deviations in the graph indicates the improper dataset. 


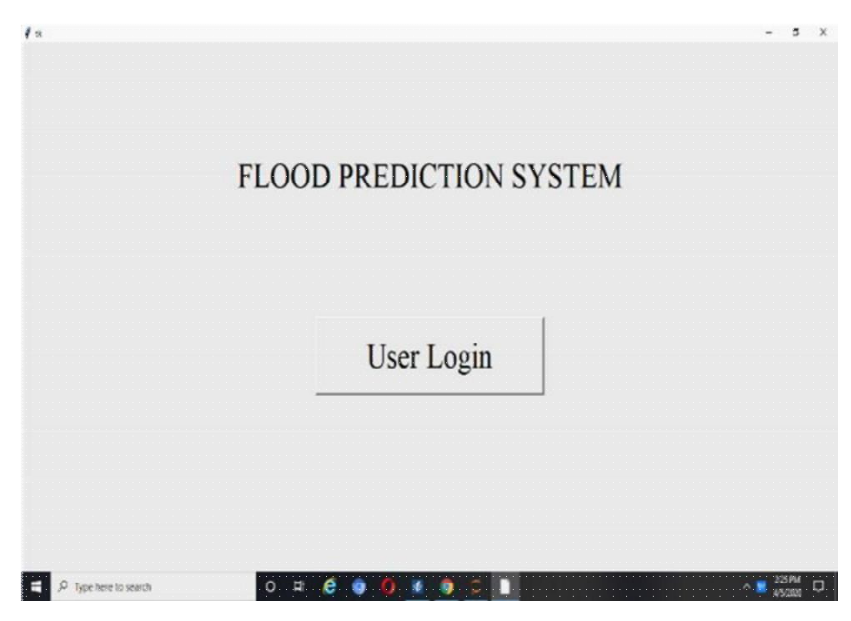

Figure 8: Login page

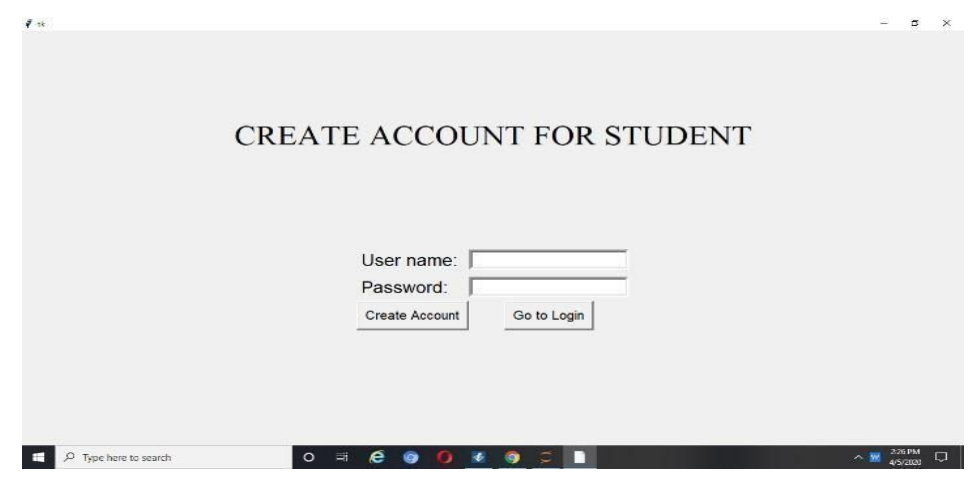

Figure 9:.Account creation

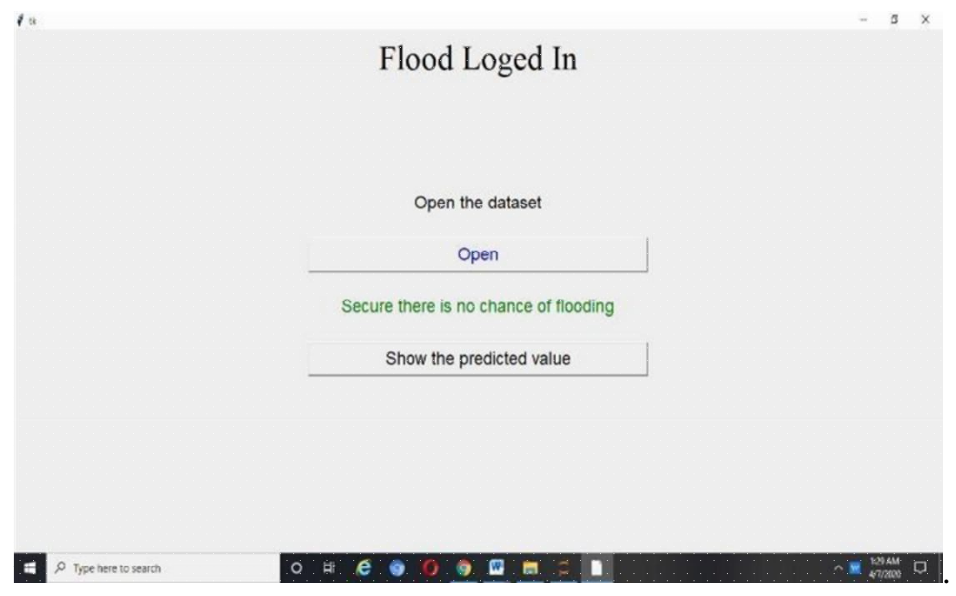

Figure 10: Flood Predicted

\section{CONCLUSION}

The development and implementation of a real time flood monitoring and forecasting system helps to accurately predict flooding. Advanced and cost efficient sensor technology is used to monitor environmental conditions. By using IoT and Machine Learning technologies it is possible to collect input information accurately and reach to the desired output.

Sensor network can be implemented in remote areas prone to flooding and its potential can be predicted from any other location. The connectivity is done through IoT technology. Real time monitoring and forecasting floods across multiple sectors can be done. Flood prediction using IoT enabled sensor network plays an essential role in enabling people to prepare for unnecessary losses. There is no need for manual recording of data to an excel format to train the model. Therefore this approach is most efficient, reliable and requires little time for prediction. Using better sensors more efficient flood prediction system can be implemented in future.

\section{REFERENCES}

[1] Flood Monitoring System (MyFMS), M.S. Baharum, R.A. Awang and N.H. Baba, 2011.

[2] Web-based platform for river flood monitoring, Riberio Alexandra, Cardoso Alberto, Marques Alfeu S., Simoes Nuno E., 2017.

[3] A. McEwen and H. Cassimally, Designing the Internet of Things, 2013.

[4] A. H. H. Ngu, M. Gutierrez, V. Metsis, and Q. Z. Sheng, IoT Middleware: A Survey on Issues and Enabling Technologies, Vol. X, no. X, pp. 120, 2016.

[5] Water Harvesting Chapter 3 - Rainfall-runoff Analysis, Food and Agriculture Organization of the United Nations Natural Resources Management and Environment Department.

[6] Swapnil Bande and Prof. Dr. Virendra V. Shete, "Smart flood disaster prediction system using IoT \& Neural Networks", 2017International Conference on Smart Technology for Smart Nation, IEEE 2017.

[7] Mohammed Khalaf, AbirJaafarHussain, Dhiya AlJumeily, Paul Fergus and Ibrahim OlatunjiIdowu "Advance Flood Detection and Notification System based on Sensor Technology and Machine Learning Algorithm".

[8] Indra Riyanto, Lestari Margatama, Angga Ariawan, Luhur Bayuaji, Mia Rizkinia, Dodi Sudiana, Harry Sudibyo 
S and Josaphat Tetuko Sri Sumantyo, "Web Camera Sensor Coupled with Lidar Data Flood Map for Flood Warning System ”.

[9] Vinodh P Vijayan, Deepti John, Merina Thomas, Neetha V Maliackal, Sara Sangeetha Varghese "Multi Agent Path Planning Approach to Dynamic Free Flight Environment",International Journal of Recent Trends in Engineering (IJRTE), ISSN 1797-9617 Volume 1, Number 1, May 2009, Page(s): 41-46.

[10]V P Vijayan, Biju Paul "Multi Objective Traffic Prediction Using Type-2 Fuzzy Logic and Ambient Intelligence" International Conference on Advances in Computer Engineering 2010, Published in IEEE Computer Society Proceedings, ISBN: 978-07695-4058-0, Print ISBN: 978-1-4244-7154-6

[11] Vijayan V P, Gopinathan E "Improving Network Coverage and Life-Time in a Cooperative Wireless mobile Sensor Network "Fourth International Conference on Advances in computing and communications (ICACC) Aug, 2014. Published in IEEE Computer Society Proceedings. Print ISBN: 978-1-4799-4364-7, INSPEC AccessionNumber:14630874,DOI:10.1109/ICACC.2014.1 6 PP 42-45. 